\title{
Synthesis and characterization of saturated polyester and nanocomposites derived from glycolyzed PET waste with varied compositions
}

\author{
SUNAIN KATOCH* $*$ VINAY SHARMA ${ }^{\dagger}$ and P P KUNDU \\ Department of Applied Chemistry, Sri Sai University, Palampur 176 061, India \\ ${ }^{\dagger}$ Department of Research and Development (Apollo Tyres), Baroda 391 760, India \\ † Department of Polymer Science and Technology, University of Calcutta, Kolkata 700 009, India
}

MS received 17 January 2012

\begin{abstract}
Saturated polyester resin, derived from the glycolysis of polyethyleneterephthalate (PET) was examined as an effective way for PET recycling. The glycolyzed PET (GPET) was reacted with the mixture of phthalic anhydride and ethylene glycol (EG) with varied compositions and their reaction kinetic were studied. During polyesterification of GPET, acid and EG, the parameters like degree of polymerization $\left(D P_{n}\right)$, extent of reaction $(p)$ acid value and hydroxyl values were measured. The thermomechanical properties and the morphologies of the saturated polyester nanocomposites were examined by using a differential scanning calorimetry (DSC), dynamic mechanical analysis (DMA), wide angle X-ray diffraction (WAXRD) and transmission electron spectroscopy (TEM). There were significant differences observed in $T_{\mathrm{g}}, T_{\mathrm{m}}$ and $T_{\mathrm{c}}$ before and after addition of GPET and clay content. Nanocomposites with lower content of organoclay showed intercalated morphology while by increasing the amount of organoclay, the exfoliated morphology was more prevalent. Water vapour transmission (WVT) was determined for saturated polyester nanocomposite sheets according to ASTM E96-80.
\end{abstract}

Keywords. Saturated polyester; mechanical properties; recycling; nanocomposites.

\section{Introduction}

Usual reactants for the saturated polyesters are a glycol and an acid or anhydride. It is the family of polyesters in which the polyester backbones are saturated and hence unreactive as compared to the more reactive and unsaturated ones. PET is one of the saturated polyester which is semicrystalline polymer, possessing an excellent chemical resistance, thermal stability and spinnability. It has been used in such diverse fields as the packaging, electrical, automotive and construction industries. PET is widely used in the packaging of beverages and drugs. The properties required in food packaging are: salability, stiffness, flexibility, barrier properties, heat resistance and transparency, but it is well known that no single polymer can satisfy all these requirements. Recent global concerns are focused on development of recycling technologies of waste plastics (Miyake 1996; Kawabata 1999). Among a variety of waste plastics, PET has been one of the most pressing environmental concerns in recent years, because of its growing demand and supply all over the world (Paszun and Spychaj 1997; Spychaj and Paszun 1998; Kishiro 1998). Plastic recycling, except incineration and landfill, is generally classified into material, thermal, and chemical recycling. Great attention is paid to chemical

\footnotetext{
*Author for correspondence (sunainkatoch@yahoo.co.in)
}

recycling, which basically means the recovery of monomers, but which can actually be used for processes, which produce interesting chemicals or intermediates from PET waste. A growing interest has been observed in the use of PET wastes for the production of specialized products such as unsaturated/saturated polyester, polyurethane, polymer composite and polymer concrete (Vaidya and Nadkarni 1987a, b, 1988). A great percentage of PET is recycled by various methods and for several applications (Campanelli et al 1993). It is known that the incorporation of a few weight percent of layered silicate enhances the properties of virgin polymer concurrently (Sinha Ray and Okamoto 2003). Also, nanocomposites of PET/layered silicate are envisaged to improve the shortcomings of PET, such as low thermal distortion temperature and low modulus of elasticity, which has limited its application as an engineering plastic. Polymer/clay nanocomposites can have two structures: (i) intercalated, where the polymer is located in the clay galleries, expanding the clay structure and retaining some long distance between the platelets and (ii) exfoliated, where the original face to face structure of the clay platelets has been destroyed and single clay sheets are randomly dispersed in the polymer (Alexandre and Dubois 2000; Sinha Ray and Okamoto 2003; Dopper et al 2004; Lee et al 2006).

In this research article, we studied the reaction kinetic during the synthesis of saturated polyester (from GPET waste). This has been done for the estimation of the maximum 
incorporation of GPET in the reaction medium in the place of EG. This GPET which contains mainly hydroxylterminated oligomers further used for the synthesis of saturated polyester and their nanocomposites. To obtain the nanocomposites without thermal degradation during the processing, a thermally stable organoclay montmorillonite is used. In this paper we outline, from a practical point of view, the state of the art of the main technology which allows us to recycle PET for value-added products.

\section{Experimental}

\subsection{Materials used}

Discarded PET bottles from soft drinks were procured from scrapers, cleaned thoroughly and cut into small pieces $(6 \times 6 \mathrm{~mm})$. Ethylene glycol (EG) and zinc acetate minimum assay 99\% were procured from E. Merck (India) Pvt. Ltd, Mumbai. Acetic anhydride (acetylating agent), pyridine, phthalic anhydride (PA) and acetone were obtained from CDH (New Delhi, India). Montmorillonite (K-10) (MMT) having surface area $270 \mathrm{~m}^{2} / \mathrm{g}$ and cetyltrimethyl ammonium bromide (CTAB), were purchased from Aldrich Chemical Company (Milwaukee, MI). All chemicals and reagents were of analytical reagent grade.

\subsection{PET pre-treatment}

The waste PET bottles were collected, sorted, washed and dried. Bottles were sorted by colour and if any undesirable parts were present, like paper labels and caps, were removed. The bottles were washed at $80{ }^{\circ} \mathrm{C}$ for $30 \mathrm{~min}$ in $2 \mathrm{wt} \% \mathrm{NaOH}$ solution, dried at room temperature, cut into small flakes $(6 \times 6)$ and dried for $24 \mathrm{~h}$ at $100{ }^{\circ} \mathrm{C}$ to eliminate moisture.

\subsection{Glycolysis of PET waste}

Five-necked glass reactor with reflux is used for the glycolysis of PET scrap. Molar ratio of PET to EG was taken 1:2, respectively. $\mathrm{Zn}\left(\mathrm{CH}_{3} \mathrm{COO}\right)_{2}$ was used as trans-esterification catalyst. The mixture was charged into a five-necked glass round-bottom flask, which was fitted with a stirrer (PTFE blade), reflux condenser, nitrogen inlet/outlet and a thermocouple linked to a temperature regulation device. The total time taken for the digestion of PET flakes is $7-8 \mathrm{~h}$ at the required temperature of $220^{\circ} \mathrm{C}$. At the end, the contents of the reaction vessel were allowed to cool at room temperature and $500 \mathrm{~mL}$ of distilled water was added while stirring vigorously. The water would dissolve all unreacted EG and the catalyst. After filtration, the residual was transferred to a beaker containing $500 \mathrm{~mL}$ of distilled water and the suspension was boiled with stirring. This was intended to extract the bis(hydroxy ethyl terephthalate) BHET, which is known to be quite soluble in boiling water (Vaidya and Nadkarni 1988).
After prolonged boiling, while still hot, it was quickly filtered. Upon immersing the filtrate in an icebath, the crystalline needles were precipitated and its melting point after filtration and drying in a vacuum oven was in the range of $106-112{ }^{\circ} \mathrm{C}$.

\subsection{Synthesis of saturated polyester from glycolyzed PET}

Initially, a reference resin (STDPET) was synthesized by direct esterification (Ravindranath and Mashelkar 1982; Yang et al 1996; Samant and Ng 1999). The PA:EG molar ratio used is $1: 1.2$ and the reaction temperature was usually $150-180^{\circ} \mathrm{C}$. The reactor was purged with argon for $15 \mathrm{~min}$ before heating was switched on. Then the content was heated under argon atmosphere at $180^{\circ} \mathrm{C}$ until approximately $15 \mathrm{~mL}$ of water was collected. Pre-polymer produced from the direct esterification reaction is gradually heated to $200{ }^{\circ} \mathrm{C}$. In this step, EG is collected as a byproduct. The overall reaction time, including the esterification and the polycondensation processes, is long and usually varies from 7 to $10 \mathrm{~h}$.

The above experiment was repeated by replacing the EG with GPET by 100:0, 80:20, 60:40, 50:50 and 40:60 per cent, respectively. The quantity of PA remains the same for all the above combinations. The nomenclature used in this work is based on the original composition of reactants (shown in table 1).

\subsection{Synthesis of saturated polyester/clay nanocomposites (in situ polymerization)}

In situ polymerization involves the dispersion and distribution of clay layers in the monomer followed by polymerization. The layered silicate is swollen within the liquid monomer or a monomer solution, so that polymer formation can occur between intercalated sheets. The saturated polyester nanocomposites were prepared by heating the desired mixture of phthalic anhydride, ethylene glycol, GPET and modified montmorillonite clay in a fivenecked reaction kettle. The modified nanofiller of a predetermined quantity was dispersed in a reaction mixture. The detailed procedure for the modification of montmorillonite is

Table 1. Detailed composition of saturated polyester and their nanocomposites with varied GPET and filler compositions.

\begin{tabular}{lccc}
\hline $\begin{array}{l}\text { Sample } \\
\text { ID }\end{array}$ & $\begin{array}{c}\text { Glycolyzed } \\
\text { PET }(\%)\end{array}$ & $\begin{array}{c}\text { Ethylene } \\
\text { glycol }(\%)\end{array}$ & $\begin{array}{c}\text { Montmorillonite } \\
\text { clay (\%) }\end{array}$ \\
\hline STDPET & 0 & 100 & 0 \\
GPET20 & 20 & 80 & 0 \\
GPET40 & 40 & 60 & 0 \\
GPET50 & 50 & 50 & 0 \\
GPET60 & 60 & 40 & 0 \\
GPET50(2) & 50 & 50 & 2 (CTAB) \\
GPET50(4) & 50 & 50 & 4 (CTAB) \\
GPET50(5) & 50 & 50 & 5(CTAB) \\
\hline
\end{tabular}


explained in reference (Katoch and Kundu 2011). The dispersion was maintained by constant mechanical stirring at $500 \mathrm{rpm}$ (overnight for proper intercalation). The mixture was heated at $120{ }^{\circ} \mathrm{C}$ for $3 \mathrm{~h}$, followed by at $150-200{ }^{\circ} \mathrm{C}$ for 3-4 h. The whole mass was transferred to an appropriate mould to produce sheet of $1 \mathrm{~mm}$ thickness. The detailed composition is reported in table 1 .

\section{Characterization}

\subsection{Acid and hydroxyl value}

The acid values ( $\mathrm{AV}$, mg KOH/g polymer) was determined by titration of $0.5 \mathrm{~g}$ sample dissolved in $25 \mathrm{~mL}$ pyridine with $0.5 \mathrm{~N} \mathrm{KOH}$ solution using phenolphthalein as an indicator. The hydroxyl value (HV) was determined following the standard method NF T 52-113.

\subsection{Water vapour transmission rate study (WVTR)}

WVT was determined according to ASTM E96-80 (ASTM standard 1989) modified by Gontard and Guilbert (1993). Water vapour transmission (WVT) was calculated according to (1):

$$
\mathrm{WVT}=\frac{W \times X}{A},
$$

where WVT is water vapour transmission $\left(\mathrm{g} \mathrm{H}_{2} \mathrm{O} \cdot \mathrm{mm} \cdot \mathrm{m}^{-2}\right)$, $x$ the average thickness of the sheet and $A$ is the permeation area.

WVTR is the mass of water vapours transmitted through a unit area in a unit time under specified conditions of temperature and humidity. WVTR was calculated according to (2):

$$
\mathrm{WVTR}=\frac{W \times X}{t \times A} .
$$

\subsection{Transmission electron microscopy (TEM)}

To prepare specimens for TEM, a small sample was microtomed using a Leica Ultracut Cryo-ultramicrotome with a diatome diamond knife at a sample temperature of $-60{ }^{\circ} \mathrm{C}$ to obtain ultrathin (about 100-200 nm) sections. The sections were transferred onto carbon-coated $\mathrm{Cu}$ grids of 200 mesh. Clay layers consisting of silicon atoms appear dark due to less electron scattering. TEM imaging was performed on a JEOL JEM 2100 operated at $100 \mathrm{kV}$ accelerating voltage.

\subsection{Differential scanning calorimetry (DSC)}

Differential scanning calorimetric studies have been carried out using a differential scanning calorimeter (Perkin Elmer Pyris 7) in the temperature range of $50-300{ }^{\circ} \mathrm{C}$ at a heating rate of $10{ }^{\circ} \mathrm{C} / \mathrm{min}$. The sample weight used is approximately $4-5 \mathrm{mg}$. The peaks are used to determine the thermal properties of the samples.

\subsection{Dynamic mechanical analysis (DMA)}

The dynamic mechanical analysis of the bulk polymers has been conducted by using a Perkin-Elmer dynamic mechanical analyser DMA Pyris-7e in a three-point bending mode with a $110 \mathrm{mN}$ static force and a $110 \mathrm{mN}$ dynamic force. Each specimen is first cooled under liquid nitrogen to about $-40{ }^{\circ} \mathrm{C}$, and then heated at $5{ }^{\circ} \mathrm{C} / \mathrm{min}$ and at a frequency of $1 \mathrm{~Hz}$ under nitrogen. The visco-elastic properties, i.e. storage modulus $E^{\prime}$ and loss modulus $E^{\prime \prime}$ are recorded as a function of the temperature. The glass transition temperature, $T_{\mathrm{g}}$, of the polymer is obtained from the peak of the $E^{\prime}$.

\subsection{FTIR spectrometry}

The FTIR spectrometry using a Perkin-Elmer RX-I spectrophotometer was used to characterize the hot water extracted glycolyzed product.

\subsection{Wide angle X-ray diffraction (WAXRD)}

Scanning intensity curves for values of $2 \theta$ ranging from $2^{\circ}$ to $10^{\circ}$ were determined by X-ray diffraction analysis (XDS 2000, Scintag Inc., USA) using powder polymeric samples. The incident $\mathrm{X}$-ray beam $(\mathrm{CuK} \alpha, 40 \mathrm{kV}, 35 \mathrm{~mA})$ was passed through a graphite filter, and pulse height discriminator to achieve further monochromatization.

\subsection{Measurement of extent of reaction and degree of polymerization}

The extent of the reaction $(p)$ and average degree of polymerization $\left(\mathrm{DP}_{n}\right)$ are represented as (Katoch et al 2009).

$$
p=\frac{C_{0}-C}{C_{0}},
$$

where $C_{0}$ is the initial concentration of carboxyl groups and $C$ is the concentration of carboxyl group at time $t$.

$$
\mathrm{DP}_{n}=\frac{1+r}{1+r-2 p r}
$$

where $r$ is the ratio of concentrations of the hydroxyl to carboxyl group and is never less than unity.

\section{Results and discussion}

\subsection{Characterization of the GPET}

The solid product obtained from the glycolysis of PET was bis(hydroxy ethyl terephthalate), BHET, with a melting point at $106-110^{\circ} \mathrm{C}$ and the IR spectrum is given in figure 1 .

The absorptions at 3400,3000-2800, 1750 and $1300 \mathrm{~cm}^{-1}$ are due to $\mathrm{OH}, \mathrm{C}-\mathrm{H}, \mathrm{C}=\mathrm{O}$ and $\mathrm{C}-\mathrm{O}$ stretching, respectively. From our experimental data, the glycolyzed products consisted of monomers BHET and higher oligomers. BHET is 


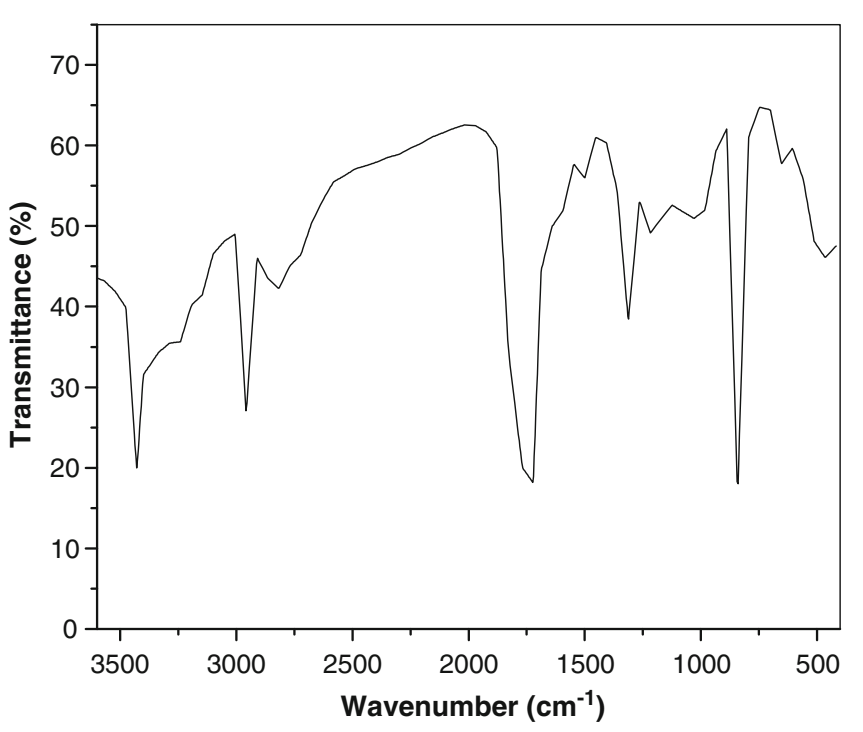

Figure 1. FTIR spectrum of BHET.

a white solid which dissolves in boiling water and was separated from the rest of oligomers. A sharp peak just below $800 \mathrm{~cm}^{-1}$ in IR spectrum is related to the $p$-substituted aromatic ring. The chemical structure of BHET is shown in scheme 1. FTIR analysis confirmed that GPET consists of hydroxyl-terminated monomer and oligomers, which can be utilized in place of glycol for the synthesis of saturated polyester.

\subsection{Characterization of saturated polyester resin}

The acid and the hydroxyl values (mg KOH/g) obtained during the synthesis of the saturated polyester (GPET-based) at different time intervals are listed in table 2. It is observed from figure 2 that for all the samples, with increase in the reaction time, acid value goes on decreasing. Probably with the progress of time, the acid groups of phthalic anhydride are consumed by glycols, leading to the formation of polyester and thus decreasing the acid value. The acid values are the maximum and minimum for the samples STDPET and GPET50, respectively. Since it is known that GPET50 sample consists of 50\% of GPET, which is mainly the mixture of hydroxyl-terminated oligomers. Obviously with an increase in hydroxyl-terminated functional groups the consumption of acid increases and resulted in the decrease in the free acid group, hence acid value decreases. It is noteworthy that incorporation of $60 \%$ GPET content in the reaction mixture resulted in an increase in the acid value. This sudden increase in the acid value is possibly due to the large changes

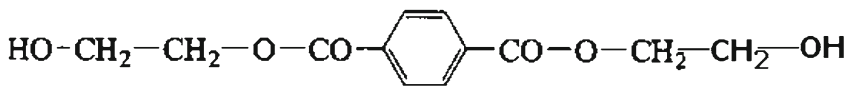

Scheme 1. Chemical structure of BHET.

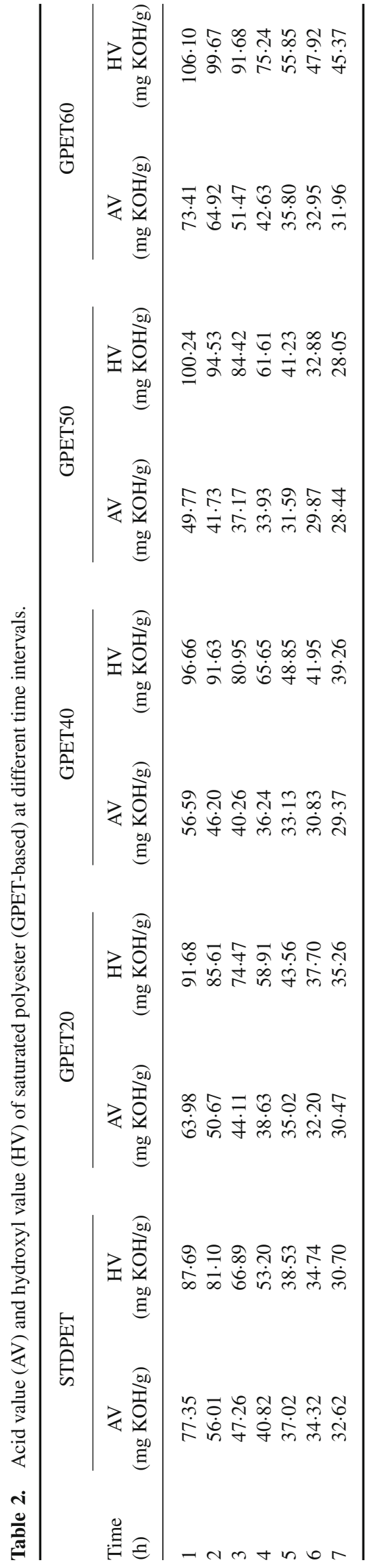




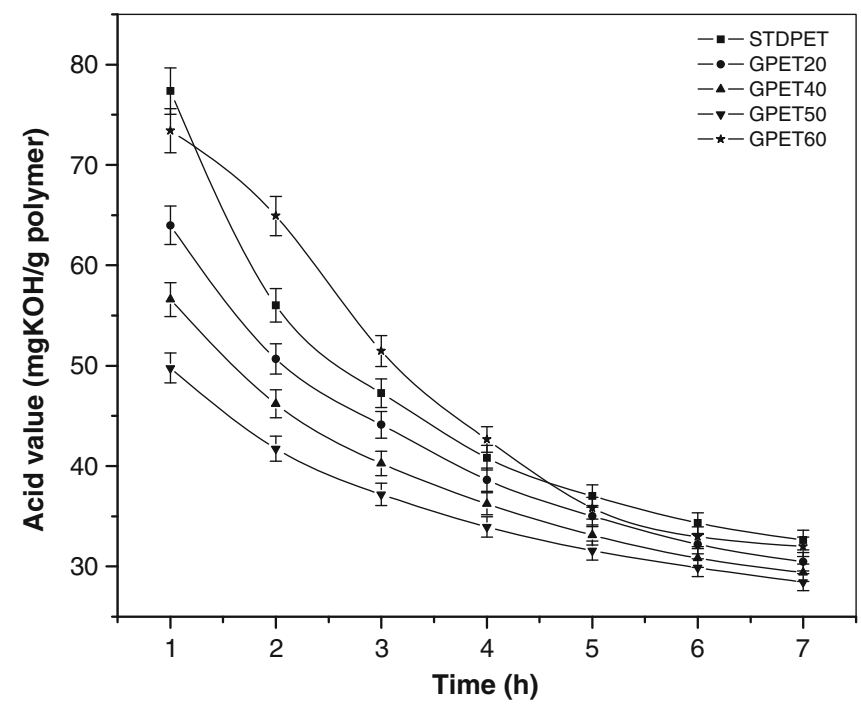

Figure 2. Acid values for the different saturated polyester resins at different time intervals.

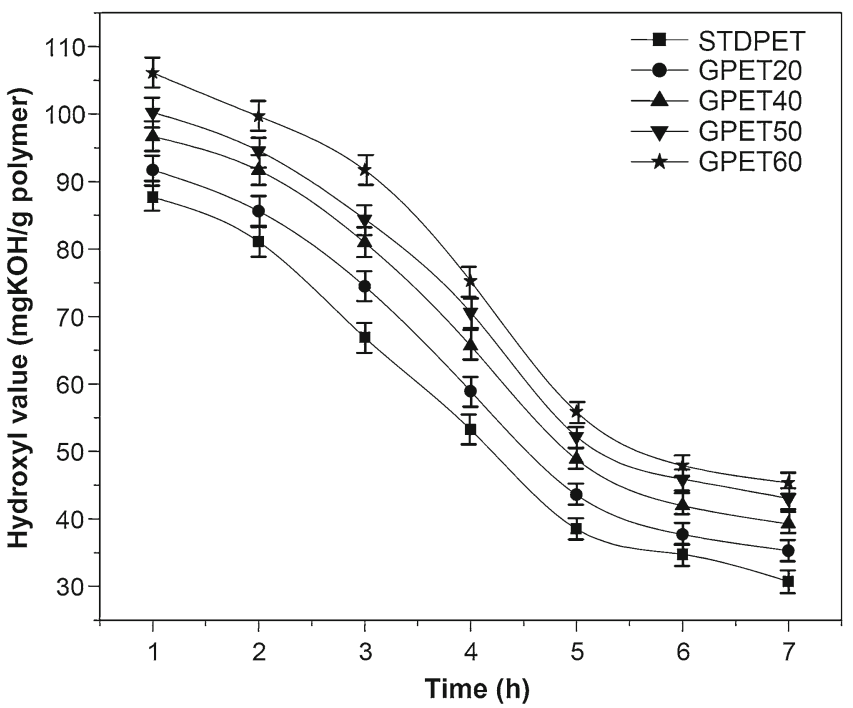

Figure 3. Hydroxyl values for the different saturated polyester resins at different time intervals.

in the polarity of the reaction mixture where the polar carboxylic acid groups are converted to the lesser polar ester linkages. The hydroxyl values for different samples during kinetic study of saturated polyester (GPET waste) are shown in figure 3.

On graphically interpreting, it was found that initially the hydroxyl value was maximum for the sample GPET60, while it was minimum for SDTPET sample. It is attributed to the higher percentage of glycolyzed PET in the reaction mixture which increases the hydroxyl value. With the progress of reaction, the hydroxyl value decreases due to the consumption of oligomers and EG by an acid. The extent of polymerization reaction is calculated according to (3) (from the acid value of table 2) and its variation with time is plotted in figure 4.

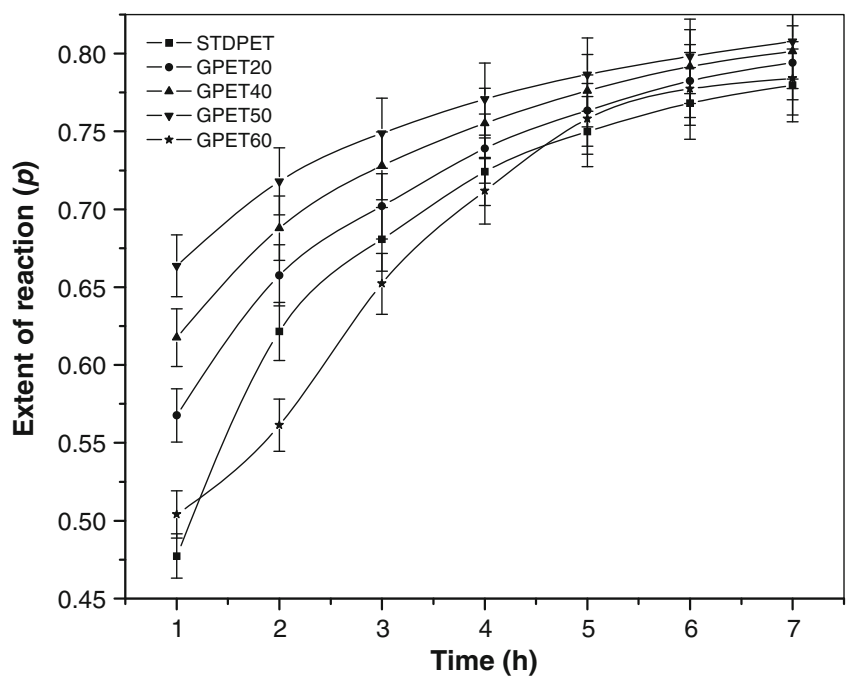

Figure 4. Plots of extent of reaction for the different saturated polyester resins at different time intervals.

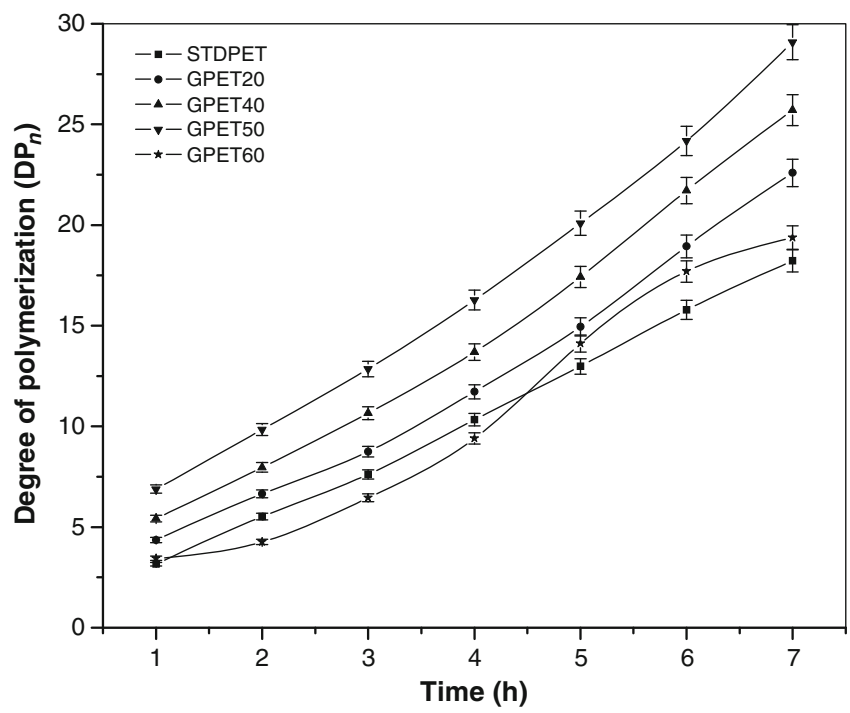

Figure 5. Degree of polymerization for the different saturated polyester resins at different time intervals.

For all samples, the extent of reaction increases with time. It is noticeable that for all the samples the extent of the reaction goes beyond $50 \%$ during the first two hours and then there is a gradual increase for the remaining hours. The extent of reaction is not even $90 \%$ after reacting for seven hours. It is evident from figure 5 that the extent of reaction is smaller for STDPET, while it is larger for the sample GPET50 during whole reaction time. The increase in the extent of the reaction with an increase in GPET is attributed to the reactive functional groups, present in the reaction medium. On comparing the value of $p$ between GPET50 and GPET60, it is higher for previous one, which is anomalous behaviour for the sample GPET60. It is due to large changes in the polarity of the reaction medium. The other factor governing the rate 
of polyesterification is the removal of water from the reaction mixture. Since polyesterification is a reversible reaction, the water should be removed in order to shift the equilibrium towards the product side. With an increase in the GPET content in the reaction medium, the molecular weight increases and so does the viscosity, which makes the removal of water from the reaction mixture difficult. Thus, the overall reaction rate of a system would depend upon the factor stated above.

The degree of polymerization is calculated from the extent of reaction ( $p$ ) and constant $r$ value (according to (4)). Although the ratio of carboxyl to hydroxyl group $(r)$ should vary with the passage of time during the reaction, it is assumed as constant for calculation of degree of polymerization $\left(\mathrm{DP}_{n}\right)$. The variations in $\mathrm{DP}_{n}$ with time for all the samples are shown in figure 5. The degree of polymerization increases with elapsed time for all the samples. This is in line with expectations, as the reacting chains are propagated to a large molecule with the passage of time. The degree of polymerization is almost following similar trend for all the samples consisting 20-50\% GPET in the reaction mixture. The decrease in $\mathrm{DP}_{n}$ for GPET60 sample may be attributed to large percentage of linear terephthalic acid moieties in the reaction medium which may retard the rate of reaction due to steric hindrance effect.

It is noteworthy that GPET50 sample is synthesized with the mixture of 50\% GPET and EG along with phthalic anhydride, whereas STDPET is synthesized by EG and TA only. So with an increase in hydroxyl terminated functional groups in the reaction mixture, acid value decreases and extent of reaction increases with the passage of time. Hence, an incorporation of 20-50\% GPET content in the reaction mixture resulted in fall in the acid value and rise in the $p$ value.

\subsection{Characterization of saturated polyester nanocomposite}

4.3a XRD: The XRD results for the MMT clay, modified clay, virgin matrix and MMT/saturated polyester nanocomposites are shown in figure 6 . The $d_{001}$ reflection for the $\mathrm{Na}^{+}-$ MMT was found at $2 \theta=8.90^{\circ}$; which corresponds to an interlayer distance of $11.90 \AA$. The XRD peak for the modified clay was found at $2 \theta=2 \cdot 86^{\circ}$; corresponding to an interlayer distance of $36.08 \AA$. As expected, the ion exchange between the clay $\left(\mathrm{Na}^{+}-\mathrm{MMT}\right)$ and the CTAB resulted in an increase in the basal interlayer spacing in comparison with pristine $\mathrm{Na}^{+}-\mathrm{MMT}$ and caused a big shift of the diffraction peak towards lower values of $2 \theta$. The organoclay peak was also observed to have $d=18.20 \AA\left(2 \theta=6.52^{\circ}\right)$; which correlated to the 002 plane of the clay layers. When the content of organoclay is increased to $2 \%$, the shift of the peak is to lower angles and subsequent increase in platelet spacing $(d=16 \cdot 25 \AA)$ suggested intercalated to partially exfoliated structure in which polyester molecules have effectively entered the distance between organoclay layers. For saturated polyester nanocomposites, a substantial decrease in the intensities of the XRD peaks was observed for clay loadings

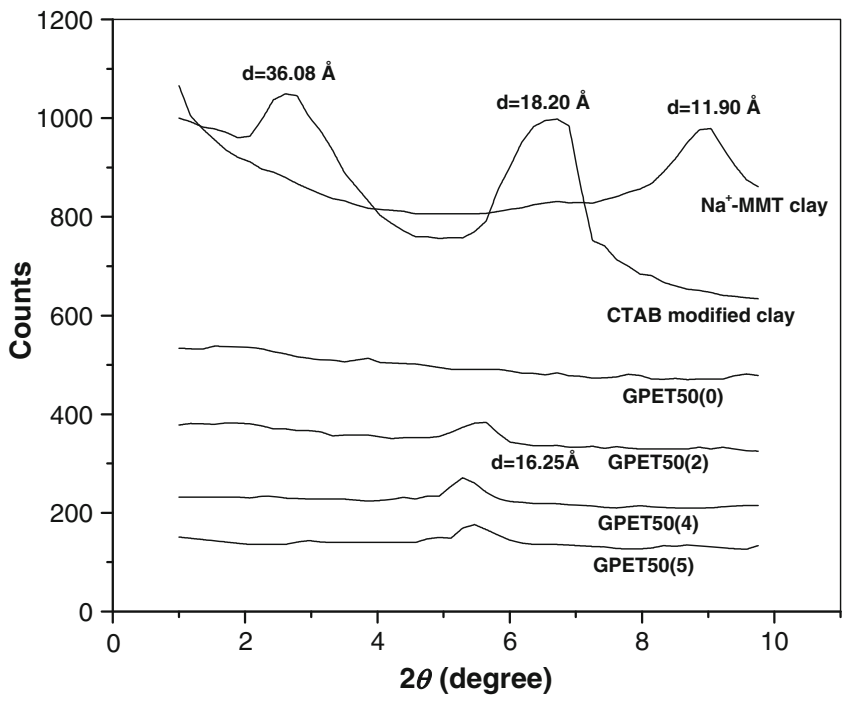

Figure 6. The XRD results for the MMT clay, modified clay, virgin matrix and MMT/saturated polyester nanocomposites.

from 2 to $5 \mathrm{wt} \%$, which suggested that the dispersion is better at a lower clay loading than at a higher clay loading.

4.3b TEM of nanocomposites: TEM images of selected nanocomposites are provided in figures $9(\mathrm{a}-\mathrm{c})$ at the scale of $100 \mathrm{~nm}$. The TEM images showed varying levels of organically modified montmorillonite (OM-MMT) dispersion and delamination in Na-MMT/saturated polyester nanocomposites at the magnification of 15,000 . Figure 7 (a) which had $2 \%$ organoclay showed many single platelets, but also contained other nanostructures. This showed that the organoclay is well dispersed in the polymer matrix at all magnification level, although some parts of agglomerated layers exist. It is observed from figure 7 (b) which has $4 \%$ organoclay; the nanocomposite has the highest degree of OM-MMT delamination and dispersion. The dispersion of Na-MMT is randomly distributed throughout the saturated polyester matrix. Here, the darker lines are clay layers and the polymer matrix is brighter. As the content of the organoclay is raised, PET molecule chains interpenetrate into the distance between organoclay layers suggesting an intercalated to partially exfoliated morphology. Figure 7(c) which consists 5\% organoclay, showed both elements of exfoliation and intercalation and also dark block (lump of dark lines, which are clay agglomeration) are clearly observed. Few aggregates are clearly visible, but due to disruption of clay tactoids mostly clay layers are well-dispersed. It is concluded that at low clay contents $(2 \mathrm{wt} \%)$, the clay particles dispersed better in the matrix without agglomeration of particles than they are for high clay contents. However, agglomerated structures become denser in the PET matrix above 4 wt $\%$ clay content. As a whole, the nanoclay has been dispersed in the polymer matrix with orientation and a few aggregates. Hence, 

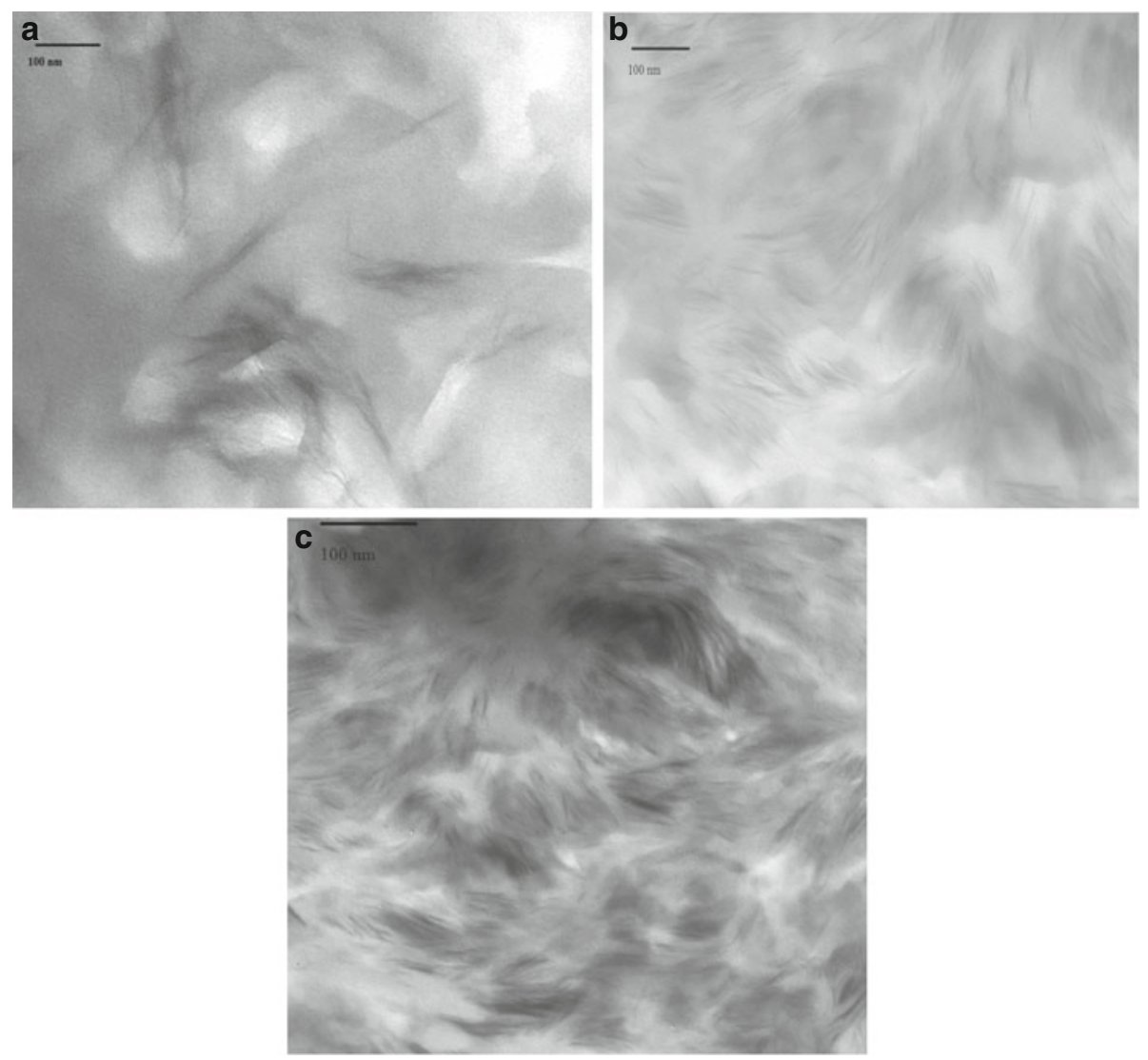

Figure 7. TEM images of saturated polyester nanocomposite at the magnification of 15,000, scale $100 \mathrm{~nm}$ for a. GPET50 containing $2 \mathrm{wt} \%$ clay, b. GPET50 containing $4 \mathrm{wt} \%$ clay and c. GPET50 containing $5 \mathrm{wt} \%$ clay.

they should probably be defined as exfoliated-intercalated nanocomposites.

4.3c WVTR study: It is the most important characterization technique for moisture sensitive products and is of great significance for package quality. It decides shelf-life of the products and is directly proportional to thickness. This property varies from polymer to polymer depending upon the thickness of the sheet. The results of water vapour transmission (WVT) of the saturated polyester sheets with varied composition of GPET and filler are shown in figure 8. The GPET and filler addition considerably decreased the WVT. It is assigned to the fact, that the incorporation of terephthalic acid moieties in the polymer chain offers better packaging, which restricts the passage of water vapours. The difference of nanostructure and the orientation of platelets could cause differences in WVT of nanocomposite sheets. Since there was no force to align clay platelets to one direction in sheet forming, so they are distributed randomly hence resist water vapour transmissions but in the stretching process (such as bottle blowing) clay platelets could align in the stretching directions. It is observed that WVT of synthesized polyester sheet, at $25^{\circ} \mathrm{C}$, varied linearly with filler percentage after $36 \mathrm{~h}$ and with very good correlation coefficient

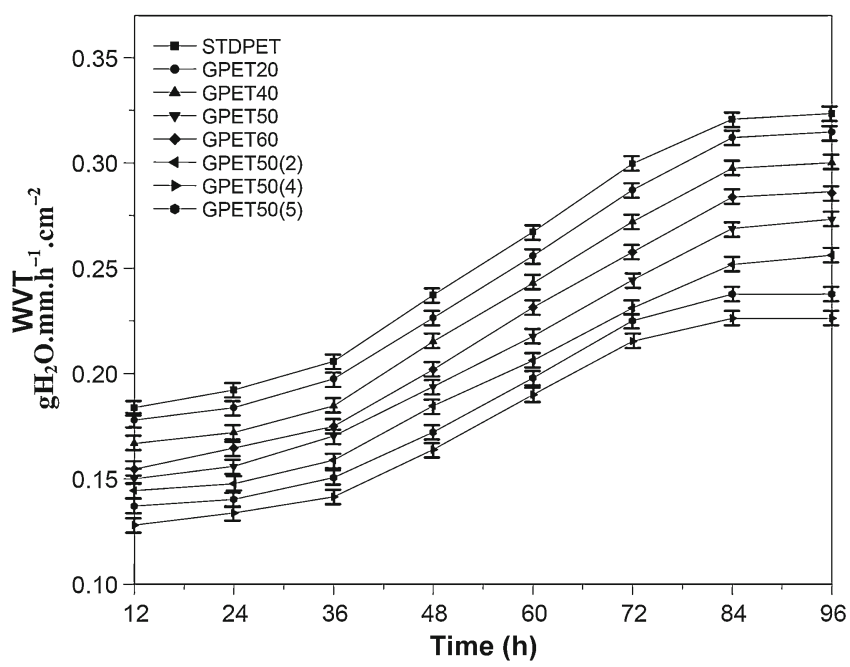

Figure 8. Water vapour transmission (WVT) of saturated polyester and their nanocomposite sheets with varied GPET and filler compositions.

$\left(R^{2}=0.95\right)$. Table 3 reported WVTR of polyester sheets. WVTR value found to be a maximum for STDPET and a minimum for GPET50(4). Hence incorporation of GPET and filler in the polymer matrix decreased the transmission rate. 
It has been confirmed from our previous manuscript (Katoch et al 2010) that diffusion coefficient of saturated polyester and their nanocomposites decreased with an increased in GPET and nanofiller content. This advocated its application in packaging material.

4.3d Differential scanning calorimetry: From the DSC curves, the glass transition temperature $\left(T_{\mathrm{g}}\right)$, the crystallization temperature $\left(T_{\mathrm{c}}\right)$ and the melting temperature $\left(T_{\mathrm{m}}\right)$ were measured. The DSC curve of the STDPET, GPET50 and three different nanocomposite samples are shown in figure 9.

The endothermic peaks of STDPET and GPET50 samples are appeared at 165 and $172{ }^{\circ} \mathrm{C}$, respectively, which are related to melting temperature. This increase in $T_{\mathrm{m}}$ is assigned to the linear terephthalic acid moieties incorporated in to the polymer backbone during synthesis of the saturated polyester. Hence, $T_{\mathrm{m}}$ is higher for GPET50 as a polymer chain contains more rigid parts and/or a polymer contains less free volume. All nanocomposites have melting temperature above $180{ }^{\circ} \mathrm{C}$. This increase in $T_{\mathrm{m}}$ could result from the fact that the platelets dispersed in the matrix could shield

Table 3. WVTR of polyester sheets with varied GPET and filler compositions.

\begin{tabular}{lcc}
\hline $\begin{array}{l}\text { Sample } \\
\text { ID }\end{array}$ & $\begin{array}{c}\text { Montmorillonite } \\
\text { clay (\%) }\end{array}$ & $\begin{array}{c}\text { WVTR } \\
\left(\mathrm{gH}_{2} \mathrm{O} \cdot \mathrm{mm} \cdot \mathrm{h}^{-1} \cdot \mathrm{cm}^{-2}\right)\end{array}$ \\
\hline STDPET & 0 & $3 \cdot 4 \times 10^{-3}$ \\
GPET20 & 0 & $3 \cdot 3 \times 10^{-3}$ \\
GPET40 & 0 & $3 \cdot 1 \times 10^{-3}$ \\
GPET50 & 0 & $2 \cdot 8 \times 10^{-3}$ \\
GPET60 & 0 & $3 \cdot 0 \times 10^{-3}$ \\
GPET50(2) & 2 & $2 \cdot 7 \times 10^{-3}$ \\
GPET50(4) & 4 & $2 \cdot 4 \times 10^{-3}$ \\
GPET50(5) & 5 & $2 \cdot 5 \times 10^{-3}$ \\
\hline
\end{tabular}

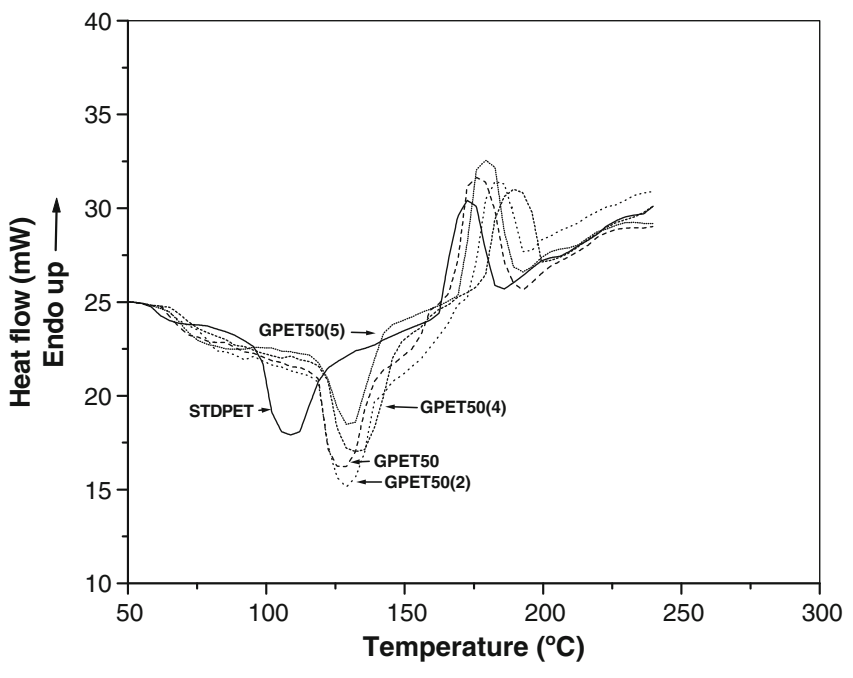

Figure 9. DSC thermogram of the saturated polyester and their nanocomposites with varied filler compositions. the conduction of heat to crystallites to some extent until at higher temperatures the heat flow is enough to melt down the crystallites.

Glass transition temperature of all nanocomposites were almost the same or only slightly higher than that of the STDPET. This increase in the $T_{\mathrm{g}}$ of all the nanocomposite samples in comparison with the STDPET can be attributed to the mixing of nanofiller. This can be explained by the existence of strong interactions between clay and the polyester matrix, which limits the movement of the polyester chain segments. This leads to an increase in the $T_{\mathrm{g}}$ of the polyester nanocomposites, which is a typical effect for the inclusion of nanofiller (montmorillonite) in a polymer system (Bandyopadhyay et al 2007). All DSC curves for nanocomposites showed a significant increase in crystallinity of nanocomposites relative to STDPET. It is known that crystallinity increases due to the addition of clay. Clay can play the role of nuclei in crystallization so that an addition of clay can increase the crystallinity of the nanocomposite. The $T_{\mathrm{g}}$, $T_{\mathrm{m}}$ and $T_{\mathrm{c}}$ obtained from DSC are reported in table 4 .

4.3e Viscoelastic properties: Polymers are viscoelastic in nature, their mechanical properties exhibit a pronounced dependence on temperature and rate of deformation. Figure $10(\mathrm{a}-\mathrm{c})$ shows the plots of storage modulus $\left(E^{\prime}\right)$, loss modulus $\left(E^{\prime \prime}\right)$ and $\tan \delta$, respectively, against temperature for saturated polyester and their nanocomposites with varied filler composition. Since, the value of $E^{\prime}$ signifies the rigidity of the material, whereas $E^{\prime \prime}$ provide information about the energy dissipated in molecular motions. The most interesting feature observed from figure 10 (a) is a sudden drop of $E^{\prime}$ for the STD sample. It became too soft as soon as it crossed the $T_{\mathrm{g}}$ whereas, the existence of $E^{\prime}$ at higher temperature for nanocomposites samples, i.e. the presence of elasticity and plasticity in the nanocomposites at higher temperature can be explained by the reinforcement effect of organoclay after glassy state (Lan and Pinnavaia 1994). It is interesting to note from figure 10(a) that there is a significant difference between the $E^{\prime}$ values of STDPET and GPET50, respectively. The higher value of the storage modulus on addition of $50 \%$ GPET in the reaction medium is attributed to the higher extent of reaction, which could be justified in figure 4 . The incorporation of terephthalic acid moieties in the polymer chain offer better packaging, which restrict the movement of molecules, hence gives the rigidity to polyester chain.

Table 4. Glass transition temperature, melting temperature and crystallization temperature obtained from DSC.

\begin{tabular}{lccc}
\hline Sample ID & $T_{\mathrm{m}}\left({ }^{\circ} \mathrm{C}\right)$ & $T_{\mathrm{g}}\left({ }^{\circ} \mathrm{C}\right)$ & $T_{\mathrm{c}}\left({ }^{\circ} \mathrm{C}\right)$ \\
\hline STDPET & 165 & 55 & 110 \\
GPET50 & 172 & 58 & 124 \\
GPET50(2) & 180 & 60 & 125 \\
GPET50(4) & 184 & 61 & 126 \\
GPET50(5) & 190 & 60 & 126 \\
\hline
\end{tabular}



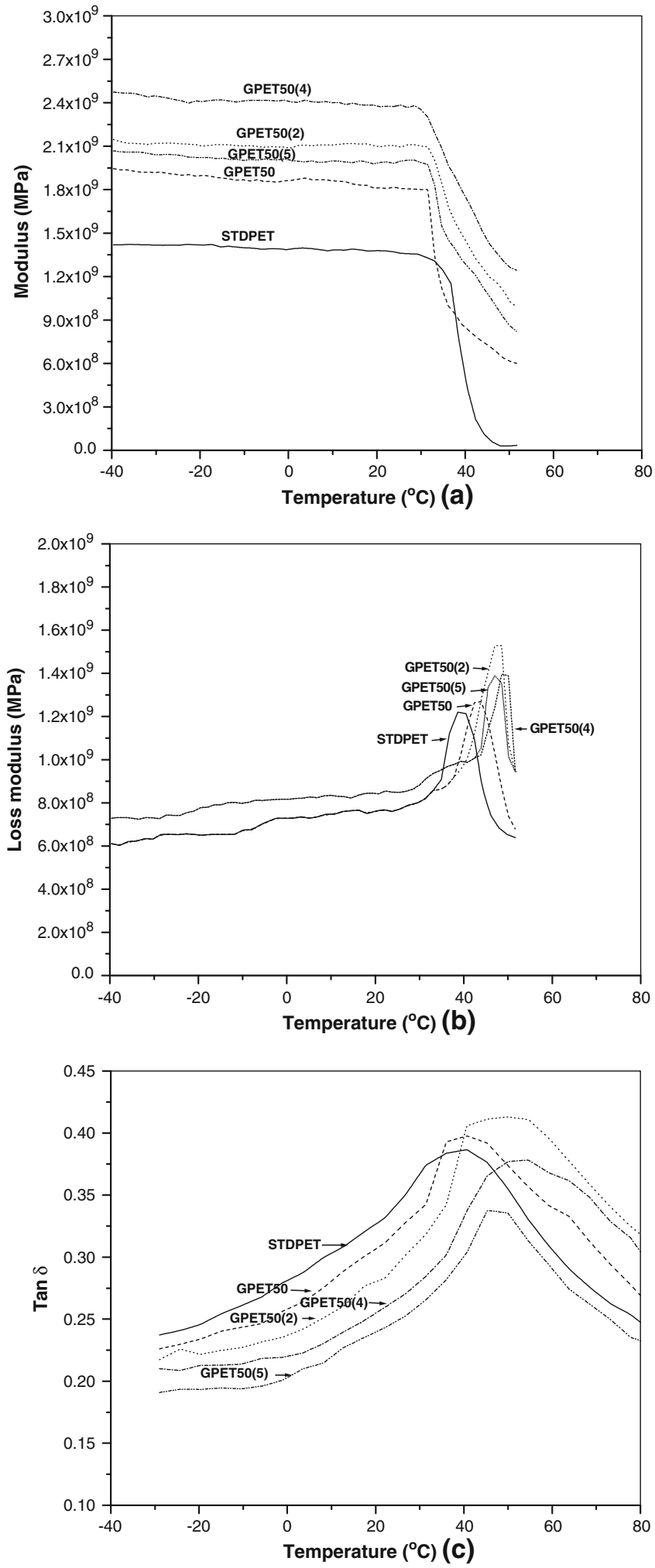

Figure 10. Temperature dependence of: (a) storage modulus; (b) loss modulus and (c) $\tan \delta$ for saturated polyester and their nanocomposites with varied filler compositions.

The minimum and maximum modulus values are observed for the sample GPET50(0) and GPET50(4). When the organoclay in polyester matrix reached $4 \mathrm{wt} \%$, the modulus
Table 5. Storage modulus, loss modulus and $T_{\mathrm{g}}$ values obtained from DMA.

\begin{tabular}{lcccc}
\hline $\begin{array}{l}\text { Sample } \\
\text { ID }\end{array}$ & $\begin{array}{c}E^{\prime} \\
(\mathrm{MPa})\end{array}$ & $\begin{array}{c}\text { Peak } E^{\prime \prime} \\
\text { value (MPa) }\end{array}$ & $\begin{array}{c}\text { Temp. range for } \\
\text { peak } E^{\prime \prime}\left({ }^{\circ} \mathrm{C}\right)\end{array}$ & $\begin{array}{c}T_{\mathrm{g}} \\
\left({ }^{\circ} \mathrm{C}\right)\end{array}$ \\
\hline STDPET & 1255 & 1220 & $34-44$ & 46 \\
GPET50 & 1800 & 1260 & $37-48$ & 48 \\
GPET50(2) & 2066 & 1390 & $39-50$ & 54 \\
GPET50(4) & 2397 & 1510 & $44-50$ & 58 \\
GPET50(5) & 1977 & 1370 & $43-50$ & 52 \\
\hline
\end{tabular}

increases about $545 \mathrm{MPa}$ over that of GPET50(0). This large increase in the modulus property of GPET50(4) owing to the presence of organoclay can be explained as follows: the amount of increase in the modulus property due to the clay layers depends on interactions between GPET molecules and layered organoclay and on the rigid nature of the clay layers. Moreover, the clay is much more rigid than the GPET molecules and does not deform or relax as much as the GPET molecules without nanofiller do. This improvement was possible because organoclay layers could be dispersed and intercalated in the GPET matrix. This is consistent with the general observation that the introduction of organoclay into a matrix polymer increases its strength and modulus (Yano et al 1997; Masenelli-Varlot et al 2002). The lower value of GPET50(5) as compared to GPET50(4) may be attributed to the tendency of clay particles to agglomerate due to poor dispersion at high clay contents, because of which the nanofiller behaves just like an ordinary filler.

The elastic and viscous components obtained by DMA analysis are reported in table 5 . The variation of loss modulus $\left(E^{\prime \prime}\right)$ with temperature is shown in figure $10(\mathrm{~b})$. It provides information about the energy dissipated in molecular motions and thereby the impact resistance of the material. Greater the area under $E^{\prime \prime}$, greater is the energy dissipation, signifying better impact strength. It is well known that peak on the loss modulus curve gives the $T_{\mathrm{g}}$ value. From the figure $10(\mathrm{~b})$, it is notified that the $T_{\mathrm{g}}$ measured by loss modulus peak for all nanocomposite samples are $10-15^{\circ}$ lower than $T_{\mathrm{g}}$ as measured by DSC. It is observed that the area under $E^{\prime \prime}$ is increasing on addition of GPET and filler, hence signifying better impact strength. From figure 10(c), the $T_{\mathrm{g}}$ values are calculated from $\tan \delta$ peak are reported in table 5 .

\section{Conclusions}

The GPET products (oligomers) obtained by the glycolysis of PET were reacted with the mixture of phthalic anhydride and EG with varied composition and their reaction kinetics were studied. During polyesterification reaction, HV, $\mathrm{DP}_{n}$ and $p$ are increased, while AV value decreased with an increase in the GPET content in the mixture. The maximum value of $\mathrm{DP}_{n}$ and $p$ is found to be 29 and $80 \%$ for 
the GPET50 sample. The well-defined order maintained up to $50 \%$ GPET content beyond it, the $\left(\mathrm{DP}_{n}\right)$ and extent of reaction follows decreasing trend with time. It is observed that WVTR significantly decreased with an increase in the GPET and filler content. It is noticeable from DSC curve that addition of GPET and nanofiller resulted into further increase in $T_{\mathrm{g}}$ ranges from 55 to $61{ }^{\circ} \mathrm{C}$ and maximum value was shown by GPET50(4). The $T_{\mathrm{m}}$ for all nanocomposites with 50\% GPET content is $10-15{ }^{\circ} \mathrm{C}$ higher as compared to the STDPET. The storage modulus and loss modulus values are observed maximum for GPET50(4) which is reported 2397 and $1510 \mathrm{MPa}$, respectively. It is observed that the area under $E^{\prime \prime}$ increased on addition of GPET and filler, hence signifying better impact strength.

The above observed properties advocated its possible application in packaging and automotive industries. It can be used as cartons to pack cake, sheet to food trays, stationary goods, carrier tape for IC chip, etc.

\section{References}

Alexandre M and Dubois P 2000 Mater. Sci. Eng. 281

ASTM Standard 1989 Standard test methods for water vapour transmission of materials. Annual book of ASTM standards. Designation E96-E80 (pp. 730-739). Philadelphia

Bandyopadhyay J, Sinha Ray S and Bousmina M 2007 J. Ind. Eng. Chem. 13614
Campanelli J R, Kamal M R and Cooper D G 1993 J. Appl. Polym. Sci. 48443

Dopper L M, Breen C and Sammon C 2004 Vibr. Spectrosc. 3527

Gontard N and Guilbert S 1993 J. Food Sci. 58206

Katoch S and Kundu P P 2011 J. Appl. Polym. Sci. 1222731

Katoch S, Sharma V, Kumar V and Kundu P P 2009 J. Polym. Eng. 29199

Katoch S, Sharma V and Kundu P P 2010 Chem. Eng. Sci. 65 4378

Kawabata N 1999 Kobunshi 48774

Kishiro O 1998 Kagaku Kogyo 511884

Lan T and Pinnavaia T 1994 J. Chem Mater. 62216

Lee W D, Im S S, Lim H M and Kim K J 2006 Polymer 481

Masenelli-Varlot K, Reynaud E, Vigier G and Varlet J 2002 J. Polym. Sci., Part B: Polym. Phys. 40272

Miyake A 1996 Eco. Ind. 1 34; CA 125 277711b

Paszun D and Spychaj T 1997 Ind. Eng. Chem. Res. 361373

Ravindranath K and Mashelkar R A 1982 Polym. Eng. Sci. 22610

Samant K D and Ng K M 1999 AIChE J. 451808

Sinha Ray S and Okamoto M 2003 Prog. Polym. Sci. 281539

Spychaj T and Paszun D 1998 Macromol. Symp. 135137

Vaidya U R and Nadkarni V M 1987a Ind. Eng. Chem. Res. 26 194

Vaidya U R and Nadkarni V M 1987b J. Appl. Polym. Sci. 34 235

Vaidya U R and Nadkarni V M 1988 J. Appl. Polym. Sci. 35775

Yang K S, An K H, Choi C N, Jin S R and Kim C Y 1996 J. Appl. Polym. Sci. 601033

Yano K, Usuki A and Okada A 1997 J. Polym. Sci. Part A: Polym. Chem. 352289 